J. Indones. Math. Soc.

Vol. 19, No. 1 (2013), pp. 15-21.

\title{
ENERGY OF COMPLEMENT OF STARS
}

\author{
P. R. HAmpiholi ${ }^{1}$, H. B. Walikar ${ }^{2}$, And B. S. Durgi ${ }^{3}$ \\ ${ }^{1}$ Department of Master of Computer Applications, \\ Gogte Institute of Technology, Udyambag, Belgaum - 590008, India \\ prhampi@yahoo.in \\ ${ }^{2}$ Department of Computer Science, Karnatak University, \\ Dharwad - 580003, India \\ walikarhb@yahoo.co.in \\ ${ }^{3}$ Department of Mathematics, KLE College of Engineering and Technology, \\ Belgaum - 590008, India \\ durgibs@yahoo.com
}

\begin{abstract}
The concept of energy of a graph was put forward by I. Gutman in (1978). The characteristic polynomial of a graph $G$ with $p$ vertices is defined as $\phi(G: \lambda)=\operatorname{det}(\lambda I-A(G)$, where $A(G)$ is the adjacency matrix of $G$ and $I$ is the unit matrix. The roots of the characteristic equation $\phi(G: \lambda)=0$, denoted by $\lambda_{1}, \lambda_{2}, \ldots, \lambda_{p}$ are the eigenvalues of $G$. The energy $E=E(G)$ of a graph $G$ is defined as

$$
E(G)=\sum_{i=1}^{p}\left|\lambda_{i}\right|
$$

The graphs with large number of edges are referred as graph representation of inorganic clusters, called as cluster graphs. In this paper we obtain the characteristic polynomial and energy of class of cluster graphs which are termed as complement of stars.
\end{abstract}

Key words and Phrases: Spectra of graphs, energy of graphs, stars, cluster graphs. 